\title{
High Variability in Functional Outcomes and Recurrences Between Contact Sports After Arthroscopic Bankart Repair: A Comparative Study of 351 Patients With a Minimum 3-Year Follow-Up
}

\author{
Luciano Andrés Rossi, Ph.D., Ignacio Tanoira, M.D., Tomás Gorodischer, M.D., \\ Ignacio Pasqualini, M.D., and Maximiliano Ranalletta, M.D.
}

Purpose: To compare return to sports, functional outcomes, and complications of a consecutive series of contact athletes with anterior glenohumeral instability treated with isolated arthroscopic Bankart repair for isolated anterior instability. Methods: Between January 2008 and December 2016, 351 competitive athletes who participated in contact or collision sports underwent isolated arthroscopic Bankart repair at our institution (rugby $\mathrm{n}=105$, soccer $\mathrm{n}=90$, martial arts $\mathrm{n}=36$ boxing $\mathrm{n}=28$, field hockey $\mathrm{n}=30$, handball $\mathrm{n}=31$, and basketball $\mathrm{n}=31$ ). Return to sports, the Rowe score, and the Athletic Shoulder Outcome Scoring System (ASOSS) score were used to assess functional outcomes. Complications also were evaluated. Results: The mean follow-up period was 66.7 months (range, 36-148 months) and the mean age of the 351 patients was 21.3 years (range, 17-30 years).Overall, 309 patients ( $88 \%$ ) were able to return to sports, and 284 (81\%) returned at the same level as before the injury. The mean time to return to sports was 5.3 months. The rate of return to sports, the level achieved by the patients, and time to return to sports varied significantly between sports. The Rowe and ASOSS scores showed statistical improvement after operation $(P<.001)$. The ASOSS score varied significantly between sports $(P<.001)$. There were 40 recurrences $(11.3 \%), 7$ complications $(2 \%)$ and 21 patients $(6 \%)$ underwent revision surgery. There was a significant difference in the rate of recurrences and revisions between the different contact sports. Conclusions: In athletes with glenohumeral instability who undergo isolated arthroscopic Bankart repair for isolated anterior instability, there is great variability in the rate of return to sport at the same level, in shoulder performance after returning to competition, and in the postoperative recurrence rates. Due to the high variability found in our study, results after arthroscopic Bankart repair in contact athletes should not be reported globally by including the different sports under the "collision or contact sports" label. Level of Evidence: Retrospective Case Series; Level of evidence, 4.

$\mathbf{T}$ here is an increased redislocation rate in collision athletes with glenohumeral instability operated with the Bankart procedure..$^{1-3}$ Nonetheless, there is no universally accepted definition of "contact" sports in the

From the Hospital Italiano de Buenos Aires, Buenos Aires, Argentina. The authors report that they have no conflicts of interest in the authorship and publication of this article. Full ICMJE author disclosure forms are available for this article online, as supplementary material.

This study was performed at the Shoulder Unit Department of Orthopaedic Surgery.

Received January 22, 2020; accepted July 8, 2020.

Address correspondence to Luciano Andrés Rossi, Ph.D., Peron 4190 (C1199ABB),Buenos Aires, Argentina.E-mail: luciano.rossi@hospitalitaliano. org.ar

(C) 2020 THE AUTHORS. Published by Elsevier Inc. on behalf of the Arthroscopy Association of North America. This is an open access article under the CC BY-NC-ND license (http://creativecommons.org/licenses/by-nc-nd/4.0/). 2666-061X/20104

https://doi.org/10.1016/j.asmr.2020.07.004 literature. The most widely used classification is the one proposed by the American Academy of Pediatrics Committee on Sports Medicine. ${ }^{4}$ In this classification, sports are categorized as contact, limited contact, or noncontact, according to the relative risk of an acute injury to the athlete during the competition. However, there is no clear dividing line between the 3 groups. This classification includes in its first category ("contact and collision sports") sports with a significant variability in terms of the frequency and intensity of the blows and the specific demand imposed on the shoulder. ${ }^{4}$ Although this is not the only classification of contact sports, in general most authors report the results encompassing the different types of sport under the name of "contact sports."

However, shoulder involvement in terms of the necessary resistance, strength, and precision to practice each of these sports is highly variable. ${ }^{4,10,11}$ This might 
lead to quite heterogeneous results between the groups in terms of functional scores and return to sport. ${ }^{4,10,11}$ Moreover, the frequency and intensity of the blows suffered by the athletes in each of these contact sports are also quite different and thus the recurrence rate is expected to significantly vary across each of these activities. ${ }^{4,10,11}$ The risk of including all contact sports under the same group within the classification is that results are generalized, thus hindering precision when it comes to explaining to the patient what the surgery expectations are as well as the expected results after it for each of these sports.

The purpose of this study was to compare return to sport rates, functional outcomes, and complications of a consecutive series of contact athletes (according to the classification of the American Academy of Pediatrics Committee on Sports Medicine) with anterior glenohumeral instability treated with isolated arthroscopic Bankart repair for isolated anterior instability. The hypothesis of our study was that due to the great heterogeneity of sports included as "contact sports," the functional outcomes and the complications of the Bankart repair in contact athletes would vary significantly.

\section{Methods}

This was a retrospective comparative study. Between January 2008 and December 2016, 448 athletes who participated in contact or collision sports underwent arthroscopic Bankart repair for isolated anterior instability at our institution. The inclusion criteria for this study were patients younger than 30 years, a minimum follow-up period of 36 months, at least 1 instability episode (defined as a subluxation or dislocation with spontaneous reduction or complete dislocation requiring a reduction), and a competitive sports level (regular sports with competitions and practices at least 2 times/ wk). ${ }^{10}$ All patients participated in contact or collision sports according to the definition given by Rice. ${ }^{4}$

Exclusion criteria were large bony Bankart lesions (bony defects of $>20 \%$ on the anteroinferior portion of the glenoid), engaging Hill-Sachs lesions, humeral avulsion of the glenohumeral ligament lesions, associated SLAP lesions, posterior labral tears, rotator cuff injuries, previous surgery on the same shoulder, patients with anterior or inferior hyperlaxity, and patients with multidirectional instability. The ethics committee of the Italian Hospital from Buenos Aires, Argentina, approved this study (institutional review board no. 00010193; protocol no. 5462), and all patients provided written informed consent to participate in this investigation.

\section{Evaluation}

Preoperative and postoperative evaluations consisted of a patient-based questionnaire and a physical examination performed by a shoulder fellow who did not participate in the surgical procedure (L.A.R. and I.P.).
Instability was evaluated with apprehension and relocation tests performed in the preoperative evaluation and in the last follow-up. Anterior hyperlaxity was defined as external rotation of $>90^{\circ}$ with arms at the side (reaching the frontal plane), and inferior laxity was determined through use of the Gagey hyperabduction test. $^{12,13}$

Radiography and magnetic resonance imaging were performed in all cases. If, during these studies, bony defects were suspected, computed tomography was ordered to evaluate the magnitude.

The Rowe score was used as a global outcome measure. ${ }^{14}$ Shoulder-dependent sports ability was measured with the Athletic Shoulder Outcome Scoring System (ASOSS). ${ }^{10}$ This scoring system measures subjective sport-specific perceptions of pain, instability, muscular strength and endurance, intensity, and proficiency level, with each point graduated and compared with the time before injury (defined as $100 \%$ ). Clinical outcome also was assessed using the minimal clinically important difference (MCID) for the Rowe score defined respectively as (1) an increase from baseline in overall Rowe of at least 9.7 points. $^{15}$

Patients were contacted by telephone and then examined at a minimum follow-up of 36 months. Patients also were asked if they had been able to practice their previous sports and if they had been able to perform them at the same level as before the dislocation. All surgery-related complications and reoperations were documented. This information was obtained from the electronic medical history of our institution.

\section{Surgical Technique}

The surgical technique for all of the cases in this series was an anterior arthroscopic stabilization performed in the lateral decubitus position with combined general endotracheal and regional anesthesia. All athletes underwent primary arthroscopic anterior glenohumeral stabilization surgery for anterior shoulder instability using a knotted anchor technique with simple sliding knots. We used in all cases biodegradable anchors with double suture. After complete liberation and release of the capsulolabral ligament beyond the 6-o'clock position, the labral edge was debrided. Then, the anterior and inferior glenoid rim and neck were abraded with a shaver. Typically, 3 anchors with no. 2 nonabsorbable sutures were placed on the cartilage edge of the glenoid surface (3.0 Bio-Corkscrews, Arthrex, Naples, FL). No patients in this series were treated with a posteriorinferior capsulolabral repair, rotator interval closure, SLAP repair, or remplissage.

\section{Rehabilitation}

A standardized postoperative physical therapy and rehabilitation program was used. The arm was supported in a sling for 4 weeks. After 1 week, supervised 


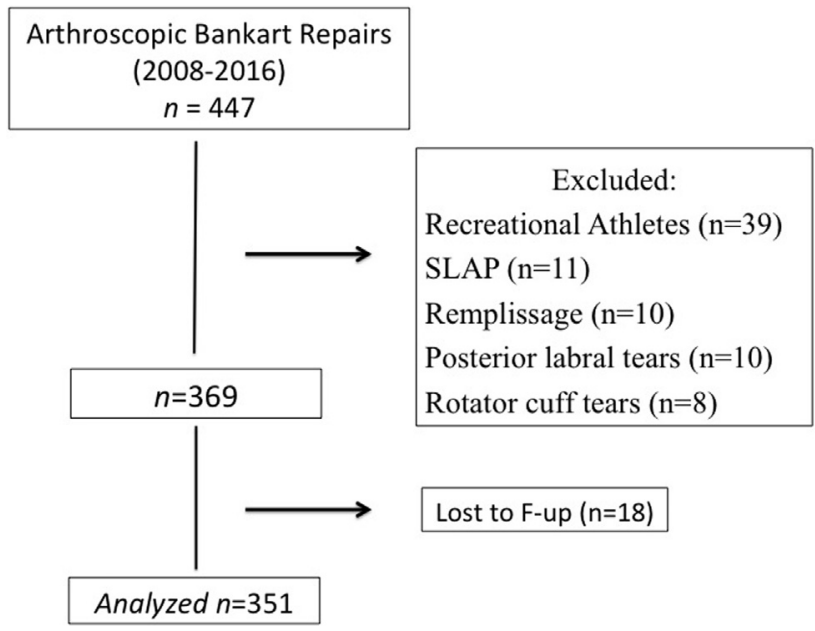

Figure 1. Flowchart demonstrating the patient-selection process. (F-up, follow-up.)

gentle physical therapy consisting of gradual passive range of motion was begun. Active-assisted range of motion exercises were started 2 weeks after surgery. When the patient could perform active forward elevation above the shoulder level, strengthening exercises were started. Running was authorized at 8 weeks. Return to sports was allowed when the patient was pain free without apprehension, full shoulder range of motion had been achieved, and shoulder strength was the near the same as before the injury.

\section{Statistical Analysis}

Preoperative and postoperative outcome scores were compared with the paired $t$ test for independent samples. Continuous variables are presented as means \pm standard deviation; categorical variables are presented as absolute and relative frequencies. Bivariate data were analyzed with a Student $t$ test, whereasl-way analysis of variance was used to analyze differences among the means of the 7 contact sports. The statistical analysis was performed with STATA, version 14 (Stata Corporation, College Station, TX). A $P$ value less than .05 was considered statistically significant

\section{Results}

Seventy-eight patients were excluded because they did not meet the inclusion criteria and 18 patients were lost to follow-up. Thus, the final analysis entailed 351 patients (95\% follow-up) (Fig 1). The mean follow-up period was 66.7 months (range, 36-148 months). There were 56 female patients $(16 \%)$ and 295 male patients $(84 \%)$ included in the study. The mean age of the 351 patients at the time of surgery was 21.3 years (range, 17-30 years). Of the 351 arthroscopic stabilizations, $202(58 \%)$ involved the dominant arm and 149 $(42 \%)$ involved the nondominant arm. The average time from the most recent instability event to surgery was 3.5 months (standard deviation, \pm 5 months). Patient demographic details for the different included sports are summarized in Table 1.

Overall, 309 patients $(88 \%)$ were able to return to sports, and $284(81 \%)$ returned at the same level as before the injury. The rate of return to sports and the level achieved by the patients varied significantly between the different contact sports. (Table 2). The mean time to return to sports was 5.3 months. Time to return to sports varied significantly between the groups from 4.5 months in field hockey and soccer players to 7.1 months in martial arts athletes $(P<.001)$ (Table 2$)$.

The Rowe score and ASSOS score showed statistical improvement after operation $(P<.001)$. Specifically, the Rowe score increased from a preoperative mean of 43.1 points to a postoperative mean of $90.1(P<.01)$. Ninety-eight percent of the athletes (345 of 351 patients) achieved a clinically significant improvement that exceeded the MCID for the Rowe score. The ASOSS score improved significantly from a preoperative mean of 53.1 to a postoperative mean of $86.8(P<$ $.001)$. The final Rowe did not change significantly between the groups at the final follow-up (Table 3). Conversely, the final ASOSS showed significantly differences between the groups at the final follow-up (Table 3). The ASOSS was greatest in the soccer and basketball groups and lowest in martial arts and boxing groups (Table 3).

There was a total of 40 recurrences $(11.3 \%)$ and 7 complications $(2 \%)$. Twenty-one patients $(6 \%)$ underwent revision surgery, all due to traumatic recurrent instability episodes during competition or training. All the patients who underwent revision were treated with a Latarjet procedure. Regarding complications, there were 3 biceps tendinitis, 2 subacromial bursitis, and 1 postoperative stiffness. All patients were treated favorably with physical therapy. There was a superficial infection of one of the arthroscopic portals in 1 patient that was treated with 2 weeks of oral antibiotics.

There was a significant difference in the rate of recurrences and revisions between the different contact sports (Table 4). The greatest recurrence rates were in rugby players and boxers $(15.8 \%$ and $20 \%$, respectively) and the lowest rates of recurrences were in soccer and field hockey players $(3.8 \%$ and $4 \%$, respectively). Similarly, the greatest revision rates were in rugby players and boxers $(11.4 \%$ and $14 \%$ respectively) and the lowest revision rates were in soccer and field hockey players ( $1 \%$ and $3 \%$, respectively).

\section{Discussion}

This study had 3 main findings. First, we found great variability in the percentage of patients who were able to return to the same level of sport they had before 
Table 1. Comparison of Demographic Characteristics Between Groups

\begin{tabular}{|c|c|c|c|c|c|c|c|c|}
\hline Variable & $\begin{array}{c}\text { Rugby } \\
(\mathrm{n}=105)\end{array}$ & $\begin{array}{l}\text { Soccer } \\
(\mathrm{n}=90)\end{array}$ & $\begin{array}{c}\text { Martial Arts } \\
(\mathrm{n}=36)\end{array}$ & $\begin{array}{l}\text { Boxing } \\
(\mathrm{n}=28)\end{array}$ & $\begin{array}{l}\text { Field Hockey } \\
\quad(\mathrm{n}=30)\end{array}$ & $\begin{array}{l}\text { Handball } \\
(\mathrm{n}=31)\end{array}$ & $\begin{array}{l}\text { Basketball } \\
(\mathrm{n}=31)\end{array}$ & $P$ Value \\
\hline$\overline{\text { Sex, male/female, } \mathrm{n}}$ & $105 / 0$ & $87 / 3$ & $30 / 6$ & $26 / 2$ & $6 / 24$ & $20 / 11$ & $21 / 10$ & $<.001$ \\
\hline $\begin{array}{l}\text { Age at time } \\
\text { of surgery, n (SD) }\end{array}$ & $20.9(4.1)$ & $21.4(4.4)$ & $21.9(3.3)$ & $22.1(3.4)$ & $21.2(4.3)$ & $20.9(3.6)$ & $21.5(3.9)$ & .691 \\
\hline $\begin{array}{l}\text { Dominant involved } \\
\mathrm{n}(\%)\end{array}$ & $60(57 \%)$ & $50(55 \%)$ & $21(58 \%)$ & $17(61 \%)$ & $\begin{array}{l}17 \\
57 \%\end{array}$ & $\begin{array}{l}19 \\
61 \%\end{array}$ & $18(58 \%)$ & .278 \\
\hline Follow-up & $67.6(22)$ & $66.4(22.1)$ & $69.1(19.7)$ & $61.1(19.9)$ & $66.2(22.2)$ & $63.8(22.3)$ & $69.6(23.1)$ & .705 \\
\hline Rowe pre, mean (SD) & $41.5(11.8)$ & $43.2(11.8)$ & $42(9.3)$ & $42.6(9.2)$ & $44.1(11.7)$ & $45.2(12.1)$ & $45.6(11.6)$ & .478 \\
\hline ASOSS pre, mean (SD) & $53.2(2.7)$ & $53.2(2.5)$ & $52.9(2.4)$ & $52.8(3.2)$ & $50.7(2.9)$ & $53.2(2.6)$ & $54.1(2.6)$ & .848 \\
\hline
\end{tabular}

ASOSS, Athletic Shoulder Outcome Scoring System; SD, standard deviation.

injury when comparing the different kinds of contact sports. Indeed, it significantly varied between $70 \%$ and $95 \%$ depending on the sport. Second, we found a significant difference in the functional scores evaluating the athlete's performance after returning to competition. Specifically, the ASOSS scores varied between $73 \%$ and $92 \%$ depending on the extent of shoulder involvement in the sport evaluated. Third, we found a significant difference in recurrence rates, which ranged from $3.8 \%$ to $20 \%$ according to the sport evaluated.

In general, the return to sport rates reported after arthroscopic Bankart repair have been high. ${ }^{1,16}$ In a recent systematic review evaluating return-to-sport rate following arthroscopic Bankart repair, Memon et al. ${ }^{16}$ reported a rate of return to a competitive level of sport of $82 \%$, whereas the rate of return to the preinjury level of competitive sports was $88 \%$. However, in the subgroup of contact athletes, the results reported by some authors have been less favorable. Cordasco et al. ${ }^{7}$ prospectively evaluated 67 contact athletes with anterior shoulder instability after arthroscopic stabilization surgery. Only $75 \%$ of the athletes returned to the same level or higher. Petrera et $\mathrm{al}^{5}$ reported a $73 \%$ rate of complete return to preinjury levels in 22 collision athletes. Cho et al. ${ }^{6}$ reported only $65 \%$ rate of complete return to preinjury levels in collision athletes. However, most authors reported global results, thus making it impossible to know the specific return to sport rates for each sport. This is an important limitation when it comes to accurately informing patients of the return to sport rate they should expect according to the kind of contact sport they practice.

In our series, although the global percentages of patients who returned to sports and who did so at the same preinjury level were high $(88 \%$ and $81 \%$, respectively), we found a significant difference between sports in terms of the percentage of patients who were able to return to the same level they had before injury, which varied between $70 \%$ and $95 \%$. For example, in sports that did not pose a significant demand on the shoulder, such as field hockey and soccer, $95 \%$ of the patients returned to sport at the same preinjury level. In contrast, in contact sports, which pose a greater demand on the shoulder, involving repetitive trauma, sprains, and blows against another competitor or against the floor as part of the sport practice (such as martial arts and boxing), we found that only $70 \%$ were able to return to the same level they had before injury. Similarly, when evaluating the time of return to sport, we found that those athletes who practiced sports with a low demand on the shoulder, such as field hockey and soccer, returned significantly earlier (4.5 months) than those practicing sports which posed a high demand on the shoulder as part of the practice, such as martial arts and overhead sports, where the average time of return to sport ranged from 6.6 to 7.1 months. This difference of approximately 2 months to return to sports was statistically significant and clinically relevant.

Regarding functional results, our study had 2 relevant findings. The first one was that shoulder performance during the sport activity varied significantly after the Bankart repair among the different contact sport athletes. The second one was that it is important to use a score that specifically evaluates shoulder function after returning to sport since although functional scores such as Rowe can be quite good, they did not always reflect shoulder performance during competition. In our study, shoulder general function

Table 2. Comparison of Sports Outcomes Between Groups

\begin{tabular}{llllllrrr}
\hline \multicolumn{1}{c}{ Variable } & Rugby & Soccer & Martial Arts & Boxing & Field Hockey & Handball & Basketball & $P$ Value \\
\hline Return to sports, \% & $89.2 \%$ & $90.5 \%$ & $85.5 \%$ & $85 \%$ & $93 \%$ & $89 \%$ & $90 \%$ & $<.004$ \\
Return same level, \% & $85.2 \%$ & $95 \%$ & $70 \%$ & $70 \%$ & $95.7 \%$ & $74.2 \%$ & $77.4 \%$ & $<.001$ \\
Time to return to sports, mo & 5.5 & 4.5 & 7.1 & 6.5 & 4.5 & 6.6 & 6.4 & $<.001$ \\
\hline
\end{tabular}


Table 3. Comparison of Functional Outcomes Between Groups

\begin{tabular}{|c|c|c|c|c|c|c|c|c|}
\hline Variable & Rugby & Soccer & Martial Arts & Boxing & Field Hockey & Handball & Basketball & $P$ Value \\
\hline Final Rowe, mean (SD) & $88.3(19.1)$ & $92.1(12.3)$ & $86.5(15.4)$ & $88.9(9.8)$ & $89.8(15.7)$ & $91.8(13.6)$ & $92.6(11.9)$ & .270 \\
\hline Final ASOSS, mean (SD) & $90.9(5.5)$ & $92.3(4.6)$ & $74.4(9.9)$ & $73.7(12.7)$ & $94.1(4.9)$ & $87.8(9.5)$ & $91.8(5.9)$ & $<.001$ \\
\hline
\end{tabular}

ASOSS, Athletic Shoulder Outcome Scoring System; SD, standard deviation.

was evaluated using the Rowe score and it was greater than 85 of 100 in all groups, which means very good general functional results. Moreover, $95 \%$ of the patients achieved a clinically significant improvement that exceeded the MCID for the Rowe scores. However, when specifically evaluating shoulder function during the sport activity with the ASOSS score, we found that the final results varied significantly between 73 and 94 depending on the force, strength, and general demand posed on the shoulder in each sport. Specifically, athletes practicing sports more demanding on the shoulder, such as martial arts and boxing, had significantly worse results in the ASOSS score than the rest of the athletes. In addition, the suboptimal results shown in the ASOSS for martial arts and boxing athletes were reflected in the lower return to sport rate at the same level before injury observed in these athletes.

Some previous works have yielded similar results. ${ }^{10}$ Stein et al. ${ }^{10}$ prospectively evaluated shoulder sportspecific impairments after arthroscopic Bankart repair in 47 athletes. Patients were analyzed separately according to the sport: noncollision/nonoverhead (Gl), collision (G2), overhead (G3), and martial arts (G4). Similar to our study, the authors found that the greater the demand on the shoulder required for practicing the sport, the worse the rate of return at the same sport level. Specifically, the G1 and G2 athletes had reachieved the preinjury sport activity and sport proficiency status with excellent ASOSS scores, whereas G3 and G4 athletes remained at an inferior activity level and proficiency level. Moreover, and in line with our study, the authors found that the results shown by the scores typically used such as, Rowe, Constant, or Walch-Duplay, did not correlate with the scores which specifically evaluated shoulder performance in the sport (ASOSS and SSAS). ${ }^{10}$ Therefore, although there is no agreement in the literature as to which score should be used to evaluate patients who underwent glenohumeral instability surgery, ${ }^{17}$ we think it is important to evaluate athletes according to a score that reflects shoulder performance during the sports practice since general scores might not accurately reflect shoulder sport performance, thus overestimating the results.

Regarding recurrences, several previous authors have reported a high risk of recurrences associated with Bankart repair in contact athletes. ${ }^{1,18,19}$ Alkaduhimi et al. $^{19}$ in a recent systematic review evaluated the redislocation risk after arthroscopic Bankart repair in collision athletes. The authors evaluated 16 studies, comparing the redislocation rate between collision and noncollision sports, and reported that collision athletes have an increased absolute risk of 8 for development of postoperative instability in comparison with noncollision athletes. However, the included studies that evaluated collision athletes reported the results globally without distinguishing between the different sports. A relevant finding in our study was the great variability found in the recurrence rates among the different contact sports. In those sports in which blows against another competitor or against the floor are frequent and a necessary part of the competition, we found greater recurrence rates. Specifically, in our study, we had a recurrence rate of $15.8 \%$ and $20 \%$ in rugby and martial arts, respectively. In contrast, in sports with infrequent contact and lower intensity such as soccer and field hockey, the recurrence rates were of only $3.8 \%$ and $4 \%$, respectively. In addition, it is important to highlight that all recurrences were due to sport trauma during training or competition. Therefore, there seems to be a clear association between intensity and frequency of the impacts and the risk of recurrences.

Recurrences are not the only complications associated with arthroscopic Bankart repair. In a recent systematic literature review, Williams et al. ${ }^{20}$ reported a rate of complications associated to the arthroscopic Bankart repair of $1.6 \%$, excluding recurrences. In our series, we obtained similar results, with a total rate of complications of $2 \%$, excluding recurrences. However, we found no significant differences between the groups in relation to this variable. Finally, with respect to revisions, in a recent systematic review of the literature, Murphy

Table 4. Comparison of Recurrences, Complications, and Revision Rates Between Groups

\begin{tabular}{|c|c|c|c|c|c|c|c|c|}
\hline Variable & Rugby & Soccer & Martial Arts & Boxing & Field Hockey & Handball & Basketball & $P$ Value \\
\hline Recurrences & $15.8 \%$ & $3.8 \%$ & $20 \%$ & $20 \%$ & $4.6 \%$ & $8.3 \%$ & $6.4 \%$ & .004 \\
\hline Complications & $2 \%$ & $1 \%$ & $2.7 \%$ & $0 \%$ & $3.3 \%$ & $3 \%$ & $3 \%$ & .986 \\
\hline Revisions & $11.4 \%$ & $1 \%$ & $11 \%$ & $14 \%$ & $3 \%$ & $6.4 \%$ & $3.2 \%$ & $<.001$ \\
\hline
\end{tabular}


et al. ${ }^{21}$ evaluated 9 studies reporting Bankart repair results with a minimum follow up period of 10 years. The authors reported a total revision rate of $17 \%$ after an average follow-up of 148 months. In our study, the final revision rate was $6 \%$. However, this rate significantly varied among groups (from $1 \%$ to $11 \%$ ), and this was due to the fact that the indication for revision was in all cases an instability recurrence. It is important to stress that even though the revision rate in our series was more favorable than that reported by Murphy et al., some patients who experienced recurrences were indicated to undergo a revision procedure and they decided not to do it. Consequently, although the revision rate was $6 \%$, the number of patients with a medical indication to undergo revision was higher.

\section{Limitations}

This work has some limitations that should be pointed out. First, it is a retrospective study and thus has all the limitations inherent to this kind of studies. Second, there was no control group operated on with another surgical technique. It would have been useful to have a control group operated on, for example, with the Latarjet technique to evaluate whether the kind of procedure used has an impact on the sport results and the complications or whether all the differences found are due to the kind of sport involved. Third, although the ASOSS score is frequently used, it is not validated in the literature. Finally, some contact sports were not analyzed because their practice is not frequent in our setting and, therefore, we did not have a sufficient number of cases to include in the analysis. However, we believe that this study has a sufficient number of patients in each of the 7 groups included to demonstrate our hypothesis.

\section{Conclusions}

In athletes with glenohumeral instability who underwent isolated arthroscopic Bankart repair for isolated anterior instability, there is great variability in the rate of return to sport at the same level, in shoulder performance after returning to competition, and in the postoperative recurrence rates. Due to the high variability found in our study, results after arthroscopic Bankart repair in contact athletes should not be reported globally by including the different sports under the "collision or contact sports" label.

\section{References}

1. Yamamoto N, Kijima H, Nagamoto H, et al. Outcome of Bankart repair in contact versus non-contact athletes. Orthop Traumatol Surg Res 2015;101:415-419.

2. Nakagawa S, Mae T, Sato S, Okimura S, Kuroda M. Risk factors for the postoperative recurrence of instability after arthroscopic Bankart repair in athletes. Orthop J Sports Med 2017;5:2325967117726494.
3. Nakagawa S, Mae T, Yoneda K, Kinugasa K, Nakamura H. Influence of glenoid defect size and bone fragment size on the clinical outcome after arthroscopic Bankart repair in male collision/contact athletes. Am J Sports Med 2017;45: 1967-1974.

4. Rice SG. American Academy of Pediatrics Committee on Sports Medicine: Recommendations for participation in competitive sports. Pediatrics 1988;81:737-739.

5. Petrera M, Dwyer T, Tsuji MR, Theodoropoulos JS. Outcomes of arthroscopic Bankart repair in collision versus noncollision athletes. Orthopedics 2013;36:e621-e626.

6. Cho NS, Hwang JC, Rhee YG. Arthroscopic stabilization in anterior shoulder instability: Collision athletes versus noncollision athletes. Arthroscopy 2006;22:947-953.

7. Cordasco FA, Lin B, Heller M, Asaro LA, Ling D, Calcei JG. Arthroscopic shoulder stabilization in the young athlete: Return to sport and revision stabilization rates. J Shoulder Elbow Surg 2019;29:946-953.

8. Leroux TS, Saltzman BM, Meyer M, et al. The influence of evidence-based surgical indications and techniques on failure rates after arthroscopic shoulder stabilization in the contact or collision athlete with anterior shoulder instability. Am J Sports Med 2017;45:1218-1225.

9. Saper MG, Milchteim C, Zondervan RL, Andrews JR, Ostrander RV 3rd. Outcomes after arthroscopic Bankart Repair in adolescent athletes participating in collision and contact sports. Orthop J Sports Med 2017;5: 2325967117697950.

10. Stein T, Linke RD, Buckup J, et al. Shoulder sport-specific impairments after arthroscopic Bankart repair: A prospective longitudinal assessment. Am J Sports Med 201 1;39: 2404-2414.

11. Kasik CS, Rosen MR, Saper MG, Zondervan RL. High rate of return to sport in adolescent athletes following anterior shoulder stabilisation: A systematic review. J ISAKOS 2019;4:33-40.

12. Gagey OJ, Gagey N. The hyperabduction test. J Bone Joint Surg Br 2001;83:69-74.

13. Coste JS, Jund S, Lemaire M, Pascal B. Evaluation prospective arthroscopique du test de laxité du ligament gléno-huméral inférieur. Rev Chir Orthop 1999;85:61.

14. Rowe C, Patel D, Southmayd W. The Bankart procedure: A long-term end-result study. J Bone Joint Surg Am 1978;60:1-16.

15. Park I, Lee JH, Hyun HS, Lee TK, Shin SJ. Minimal clinically important differences in Rowe and Western Ontario Shoulder Instability Index scores after arthroscopic repair of anterior shoulder instability. J Shoulder Elbow Surg 2018;27:579-584.

16. Memon M, Kay J, Cadet ER, Shahsavar S, Simunovic N, Ayeni OR. Return to sport following arthroscopic Bankart repair: A systematic review. J Shoulder Elbow Surg 2018;27:1342-1347.

17. Lukenchuk J, Sims LA, Shin JJ. Variability in outcome reporting for operatively managed anterior glenohumeral instability: A systematic review. Arthroscopy 2017;33: 477-483.

18. Torrance E, Clarke CJ, Monga P, Funk L, Walton MJ. Recurrence after arthroscopic labral repair for traumatic anterior instability in adolescent rugby and contact athletes. Am J Sports Med 2018;46:2969-2974. 
19. Alkaduhimi H, van der Linde JA, Willigenburg NW, et al. Redislocation risk after an arthroscopic Bankart procedure in collision athletes: A systematic review. J Shoulder Elbow Surg 2016:25:1549-1558.

20. Williams HLM, Evans JP, Furness ND, Smith CD. It's not all about redislocation: A systematic review of complications after anterior shoulder stabilization surgery. Am J Sports Med 2019;47:3277-3283.

21. Murphy AI, Hurley ET, Hurley DJ, Pauzenberger L, Mullett H. Long-term outcomes of the arthroscopic Bankart repair: A systematic review of studies at 10-year follow-up. J Shoulder Elbow Surg 2019;28:2084-2089. 